including demographics, clinical characteristics, infection screening, drug initiations, clinical review visits and disease activity scores. Eligible cases for audit require a record of drug start date and baseline visit. Algorithmic analysis identifies most relevant review visit and associated disease score if recorded (time-windows: post-induction, 8-16 wks; 12-month review, 44-60 wks). The rolling audit focuses on seven key performance indicators (KPIs). Cumulative results are presented, focused on each patient's first biologic initiation (April 2016 - Present).

Results 3,617 eligible cases (CD: 61\%; UC: 35\%; IBD-U: $3 \%)$. Humira 36\%; Remsima 24\%; Inflectra 18\%; Vedolizumab 14\%; Remicade 3\%; Golimumab 2\%; Ustekinumab 2\%; Not specified $1 \%$.

Table 1 shows mean KPIs (\%) across all sites, and sub-divided by eligible cases. Across the seven KPIs, 20-45\% of hospitals had results below the registry-wide mean value (arbitrary benchmark).

Conclusions The UK IBD Registry is supporting a growing network of hospitals with participation in continuous biologics audit, providing benchmarking reports to drive local and registry-wide quality improvement. Although incomplete case ascertainment and missing data are inevitable challenges, the biologics data is maturing as sites establish live registers. Results highlight an ongoing need for most centres to improve biologics monitoring through better-organised and documented review visits with objective recording of standardised outcomes.

\section{PTH-127 THE LIVED EXPERIENCES OF BRITISH SOUTH ASIAN WOMEN WITH INFLAMMATORY BOWEL DISEASE: A QUALITATIVE STUDY}

${ }^{1}$ Angela Ford, ${ }^{1}$ Hiliary Paniagua, ${ }^{2}$ Matthew Brookes, ${ }^{2}$ Helen Steed*, 'Satvinder Purewal. ${ }^{1}$ The University of Wolverhampton, Wolverhampton, UK; ${ }^{2}$ The Royal Wolverhampton NHS Trust, Wolverhampton, UK

\subsection{6/gutjnl-2019-BSGAbstracts. 186}

Introduction The rates of inflammatory bowel disease (IBD) amongst the British South Asian (SA) populations is growing, with some evidence indicating British SA are disproportionally more likely to suffer from IBD than White European. There is a limited understanding of the experience of SA women with IBD, where high rates of infertility, voluntary childlessness and fewer children are common compared to the general population. The objective of this study is to explore how South Asian women with IBD experience living with their chronic illness within dominant SA culture, where childbearing and parenthood are considered culturally mandatory.

Methods A qualitative methodology was adopted using Critical Discursive Psychology (CDP). A total of eight SA women, aged between 19-50, were recruited through two IBD clinics in West Midlands hospitals. Unstructured one to one interviews were audio recorded and analysed using CDP methods. Results Data analysis revealed that SA culture and IBD is seen as a hindrance to female worth, marriage and parenthood prospects. Further, there is a limited awareness of IBD that leads to secrecy and stigmatisation. Many SA women find coping with IBD difficult due to the perceived stigma regarding IBD and 'disease', lack of understanding about IBD (by patients before diagnosis, family and wider community), pressures regarding cultural mandatory marriage and parenthood, and the role of food (particularly spicy food) in health and illness.

Conclusion This study has shown how IBD in SA women create significant personal and social challenges, which deviates from normative expectations of women in this community. It has raised awareness of the emotional and social impact of IBD, which is critical for service provision that suitably meets the needs of this population. This study has highlighted the need for sensitive and culturally-appropriate intervention for SA patients with IBD.

\section{PTH-128 CORRELATION OF VEDOLIZUMAB TROUGH LEVELS WITH CLINICAL AND BIOCHEMICAL MARKERS IN INFLAMMATORY BOWEL DISEASE}

${ }^{1}$ Aravind Gokul Tamilarasan*, ${ }^{1}$ Antonio Guerrero, ${ }^{1}$ Laura Arias, ${ }^{1}$ Megan Burns, ${ }^{2} Z$ Zehra Arkir, ${ }^{1}$ Peter Irving. 'Guy's and St Thomas' NHS Foundation Trust, London, UK; ' ${ }^{2}$ Viapath Laboratories, London, UK

\subsection{6/gutjnl-2019-BSGAbstracts. 187}

Introduction The clinical utility of vedolizumab (VDZ) trough levels (VTLs) is not well established. The aim of this study is to determine if there is a correlation between VTLs and clinical and biochemical outcome.

Methods We performed a prospective, cross-sectional study to examine the association between VTLs and clinical and biochemical outcomes. VTLs immediately prior to VDZ infusion were collected simultaneously with CRP and Harvey Bradshaw index (HBI)/Simple Clinical Colitis Activity index (SCCAI) (for Crohn's disease, CD, and ulcerative colitis, UC, respectively). Biochemical remission was defined as CRP $\leq 5 \mathrm{mg} / \mathrm{L}$ and clinical remission was defined as $\mathrm{HBI} \leq 4$ or SCCAI $\leq 2$. Combined remission was defined as those meeting criteria for both

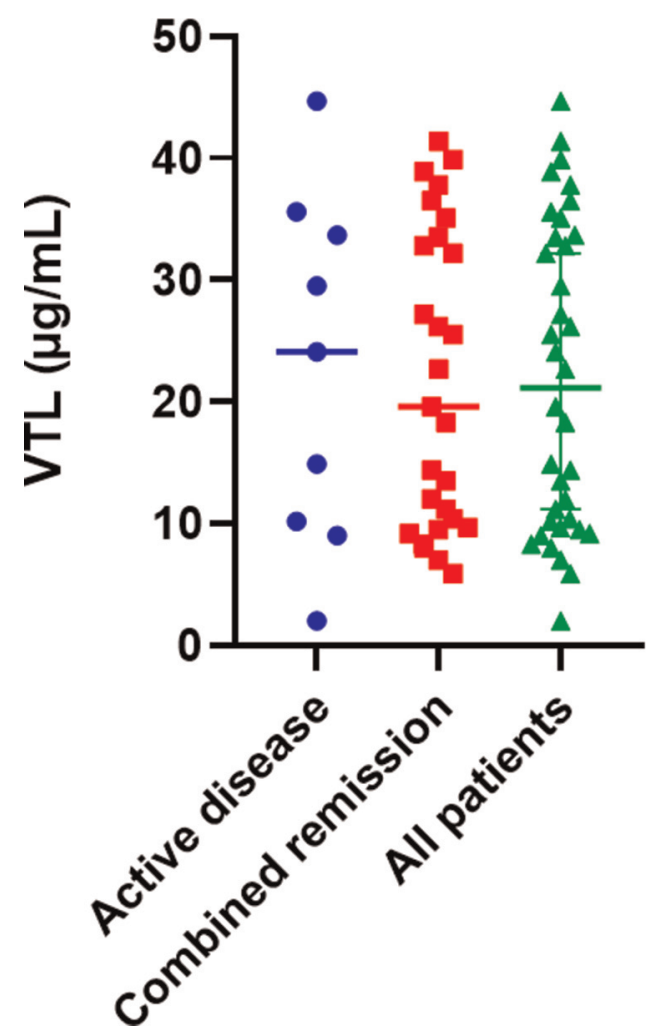

Abstract PTH-128 Figure 1 Combined remission graph 
clinical and biochemical remission. Fishers exact and MannWhitney $\mathrm{U}$ were used to compare groups and ROC analysis to identify a therapeutic threshold.

Results 37 samples with matched clinical and biochemical data were collected for 36 patients (18 UC, 14 CD and 4 undifferentiated inflammatory bowel disease). An equal number of patients had 4-weekly VDZ infusions compared to 8-weekly. 21 out of 36 patients (58\%) were on concomitant immunomodulation. The median trough level was $21.2 \mu \mathrm{g} / \mathrm{mL}$ (range $<2-44.7 \mu \mathrm{g} / \mathrm{mL}$ ) and anti-VDZ antibodies were not detected in any patient. No significant difference could be detected between median VTLs for active disease vs combined remission (24.1 vs $19.6, \mathrm{p}=0.89$ ). ROC analysis did not identify an optimal therapeutic threshold to achieve combined remission [AUC (95\% CI) $0.52(0.29-0.75)$ ]. A difference in median VTLs could not be found in the biochemical or clinical remission subgroups when compared to active disease. Similarly, a difference could not be detected when examining CD and UC subgroups. A comparison (using Fisher's exact test) of the highest vs lowest VTL quartiles did not show a significant difference in the proportion of patients who were in remission vs active disease.

Conclusions A correlation between VTLs and clinical and biochemical markers of disease activity was not able to be shown. This is a preliminary, small sample size study and analysis of VTLs for our entire VDZ cohort is being conducted at our centre.

\section{PTH-129 SIGNIFICANT COST SAVINGS UTILISING A REGIONAL PRESCRIBING APPROACH TO MESALAZINE THERAPY IN INFLAMMATORY BOWEL DISEASE}

${ }^{1}$ Jennifer Tham*, ${ }^{2}$ Tracey Sharp, ${ }^{3}$ Paul Ryan, ${ }^{3}$ Ross Pentney, ${ }^{4}$ David Watts, ${ }^{1} J o h n$ Morris. ${ }^{1}$ Glasgow Royal Infirmary, NHS Greater Glasgow and Clyde; ${ }^{2}$ West of Scotland Regional Planning; ${ }^{3}$ National Procurement, NHS National Servies UK; ${ }^{4}$ Forth Valley Royal Hospital, NHS Forth Valley

\subsection{6/gutjnl-2019-BSGAbstracts. 188}

Introduction The West of Scotland (WoS) Gastroenterology Prescribing Subgroup is a clinically led multi-disciplinary group supporting the effective prescribing of GI medicines in the five health boards $(\mathrm{HB})$ in the WoS with a population of 2.78 million. Group membership includes gastroenterologists, pharmacists, IBD nurses, with Regional Planning and National Services Scotland representation. The group builds regional consensus on an agent of choice in defined clinical indications where there are a number of therapeutic options. In Inflammatory bowel disease (IBD) the British National Formulary states there is no difference in efficacy between different oral mesalazine brands (5-ASA).

Methods We identified the opportunity to negotiate a preferential price of 5-ASA and to recommend this as the 1st line agent in patients initiating 5-ASA therapy. National Procurement Scotland, undertook a price review of the different mesalazine brands on behalf of the WoS Group. For products included in consideration, the recommended 1st line agent was the product with the lowest medicine cost to NHS Scotland in primary care, based on maintenance doses agreed by the WoS Gastro Group. In addition we recommended a switch from Asacol to Octasa in patients on maintenance 5-ASA therapy. Post guidance prescribing data was obtained from Information Services Directorate, Scotland.

Results WoS guidance recommended Salofalk preparations at 3 $\mathrm{g} / 1.5 \mathrm{~g}$ for induction and maintenance of remission for new patients with IBD. From May 2017 the guidance was disseminated to secondary and primary care via clinical advisory channels, therapeutics committee and pharmacists.

In the first year, uptake of the new guidance across the five $\mathrm{HB}$ was median 40\% (range $17-50 \%$ ) patients initiated on Salofalk compared to other mesalazine brands. From May 2017 to September 2018 there has been a total cost avoidance of $£ 109,618$ through initiating new patients on Salofalk increasing sequentially. These savings are highlighted in figure 1 .

With respect to cost savings with switching Asacal to Octasa, two HBs were excluded as they had already implemented a switch since 2016. In the three remaining HBs, switches were achieved in 71,81 and $92 \%$ of patients respectively, incurring a saving of $£ 143,633$.

Conclusions Our data shows a regional approach to cost effective prescribing of 5 ASA in IBD has been successful both in terms of implementation of the guidance and resulted in cost savings to NHS WoS of over $£ 0.25 \mathrm{M}$.

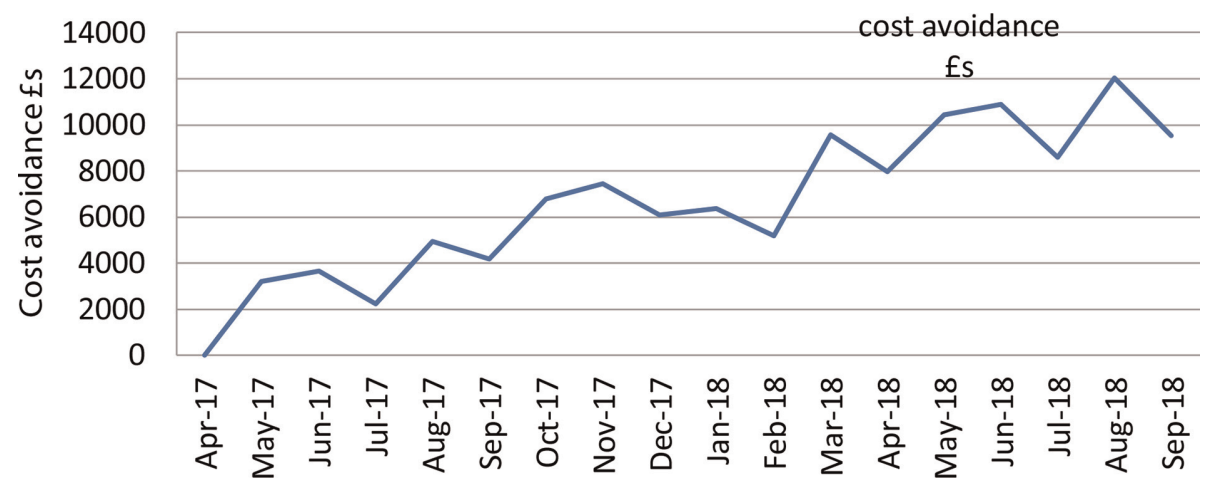

Abstract PTH-129 Figure 1 Cost avoidance associated with initiating patients on Salofalk for the period May 2017-Sept 2018 